\title{
Vlasov solver for longitudinal dynamics in beam delivery systems for x-ray free electron lasers
}

\author{
Marco Venturini, ${ }^{*}$ Robert Warnock, ${ }^{\dagger}$ and Alexander Zholents \\ Lawrence Berkeley National Laboratory, University of California, Berkeley, California 94720, USA
}

(Received 9 April 2007; published 10 May 2007)

\begin{abstract}
Direct numerical methods for solving the Vlasov equation offer some advantages over macroparticle simulations, as they do not suffer from the consequences of the statistical fluctuations inherent in using a number of macroparticles smaller than the bunch population. Unfortunately, these methods are more time consuming and generally considered impractical in a full 6D phase space. However, in a lower-dimension phase space they may become attractive if the beam dynamics is sensitive to the presence of small chargedensity fluctuations and a high resolution is needed. In this paper we present a 2D Vlasov solver for studying the longitudinal beam dynamics in single-pass systems of interest for x-ray FELs, where characterization of the microbunching instability stemming from self-field amplified noise is of particular relevance.
\end{abstract}

DOI: 10.1103/PhysRevSTAB.10.054403

PACS numbers: 29.27.Bd, 41.60.Ap, 41.60.Cr, 52.65.Ff

\section{INTRODUCTION}

Lasing in an x-ray free electron laser (FEL) critically depends on electron-beam quality. Unfortunately, a number of effects can spoil transverse emittance and energy spread as the electron beams are accelerated and compressed before entering the undulator. Of particular concern is the development of microbunching instabilities [15] stemming from the unavoidable irregularities present in the charge density at injection. Because of self-fields from radiation or space-charge, these irregularities may create energy fluctuations, which in turn feed further lumping in the charge density as the beam travels through a dispersive region. Minimizing the development of such instabilities is a significant part in the effort of designing an X-FEL.

Modeling of beam dynamics is currently carried out using a combination of macroparticle simulations and semianalytical studies of the solutions of the linearized Vlasov equation. While these tools are essential they present some well-known limitations. Macroparticle simulations are vulnerable to statistical fluctuations resulting from a bunch population smaller than the actual number of electrons. This noise can be somewhat controlled by a judicious choice of suitable filters [4] but it remains difficult to separate from the genuinely physical fluctuations that one intends to study. On the other hand, the linearized Vlasov equation fails to capture nonlinear saturation, which can be important.

A possible third approach, immune to these limitations, is to solve numerically the complete Vlasov equation. While direct numerical methods for the Vlasov equations have enjoyed a certain degree of popularity in plasma physics after the seminal paper by Cheng and Knorr [6], they have yet to find widespread application in beam

\footnotetext{
*Electronic address: mventurini@lbl.gov

${ }^{\dagger}$ Also at Stanford Linear Accelerator Center, Stanford University, Stanford, CA 94309.
}

physics [7-13]. To some extent this is a consequence of the larger computational cost: indeed, at present an application to a $6 \mathrm{D}$ phase space would be prohibitive whereas application to 4D appears feasible only using parallel computing [14-16]. The use of these methods in 2D phase space has been very rewarding, however, which encourages further efforts to develop solution technology both in more demanding 2D contexts and in higher dimensions.

In this paper we discuss a scheme for a Vlasov solver in the $2 \mathrm{D}$ longitudinal phase space suitable for applications in single-pass systems of interest for $\mathrm{x}$-ray FELs.

While a more complete treatment of the relevant physics would require at least a $4 \mathrm{D}$ phase space to fully describe the coupling between horizontal and longitudinal motion, a 2D solver is already physically significant for some applications and shares some of the algorithmic challenges with solvers in higher dimensions. Indeed the experience gained from this study will be very useful for building a 4D solver, which is in our plans for the future.

Modeling of a beam for FEL applications has to face the presence of a close correlation between longitudinal position and energy, which is deliberately added to achieve compression in magnetic chicanes. This correlation makes the simple method [9-11] of gridding the phase space on a static rectangular mesh ill suited. Two distinct strategies can be devised involving either grid adaptation or a suitable coordinate transformation. A combination of the two might also be profitable. With regard to the first, recent work by Sonnendrücker and co-workers [17] using multiscale resolution and moving grids is promising, while grid-free methods proposed in [18] may be worthy of further exploration. Concerning the second strategy a change of variables to the "interaction picture" was proposed in [13]. In the present paper we explore a method that involves a transformation to new dynamical variables that is different from the one suggested in [13], and uses cell-size adaptation in a Cartesian grid that avoids the complications of local mesh refinement. The idea for the coordinate trans- 
formation is simply to subtract the energy-position correlation from the energy coordinate, whereas the grid adaptation follows the natural beam compression occurring as the beam travels through a magnetic chicane.

In Sec. II we offer a derivation for a 2D Vlasov equation for beams with vanishing transverse emittance. We give a description of the integration method in Sec. III, and a schematic summary of the algorithm in Sec. IV. In Sec. V we illustrate an application of the Vlasov solver to the determination of the gain function for the microbunching instability and make contact with linear theory. Finally, in Sec. VI we present a tentative model for the impact of a finite horizontal emittance on microbunching and, again, make comparison with linear theory.

\section{EQUATIONS OF MOTION FOR BEAMS IN BUNCH COMPRESSORS AND ACCELERATING CAVITIES}

A beam delivery system for $\mathrm{x}$-ray FELs consists essentially of accelerating structures and magnetic chicanes for beam compression.

In a bunch compressor we use the following model of a Hamiltonian for the horizontal and longitudinal particle dynamics:

$$
H=\frac{1}{2}\left(p_{x}^{2}+k_{x} x^{2}\right)-\frac{x}{R} \delta-\frac{1}{p_{0}} \int_{-\infty}^{z} F\left(z^{\prime}, s\right) d z^{\prime}
$$

with the last term on the right-hand side (RHS) accounting for collective effects. In the above equation $k_{x}=k_{x}(s)$ is the focusing function, $R=R(s)$ the local radius of curvature of the design orbit, $\delta=\left(p-p_{0}\right) / p_{0}$ an electron relative energy deviation from the design energy $p_{0}$ [19], and $z$ the longitudinal coordinate, assumed to be positive for particles in the head of the bunch. In writing the above Hamiltonian, we neglected the term proportional to $\delta^{2} / \gamma^{2}$ (with $\gamma$ being the relativistic factor). That is, under the ultrarelativistic assumption $\gamma \gg 1$, we neglect particle slippage in $z$ not due to dispersive effects.

The collective longitudinal force $F(z, s)$ acting on an electron at $z$ within a bunch depends on the component of the electric field parallel to the particle trajectory at that point. Here, $z$ is the longitudinal distance of the electron from a synchronous particle following the design orbit. In our convention $z>0$ indicates a particle closer to the bunch head. If we denote with $w\left(z-z^{\prime}\right)$ the longitudinal component of the electric field at $z$ generated by a positive unit charge placed at $z^{\prime}$, then the longitudinal electric field $E_{s}(z, s)$ experienced by an electron at $z$ is

$$
E_{s}(z, s)=-e N \int_{-\infty}^{\infty} d z^{\prime} w\left(z-z^{\prime}\right) \rho\left(z^{\prime}, s\right),
$$

where $N$ is the number of electrons in the bunch, $-e$ their charge, and $\rho(z, s)$ the longitudinal beam density normalized to unity $\int_{-\infty}^{\infty} d z \rho(z, s)=1$.
In the case of the field due to synchrotron radiation, this formula is not strictly correct, even in the one-dimensional model pursued here. The radiation depends on the bunch form at past times, whereas the formula purports that it depends only on the form at the present time. A formalism to correct this defect, allowing bunch deformation at retarded times, was presented in [20]. A numerical test indicated that the effect of deformation is tolerably small, but more work needs to be done since the test was not made under conditions of severe bunch instability [21].

Adopting the convention that $E_{s}$ is positive if pointing in the direction of increasing $s$, the energy change per unit length induced on a particle by the electric field is

$$
\frac{d \delta}{d s}=-\frac{e E_{s}(z, s)}{p_{0}}=\frac{e^{2} N}{p_{0}} \int_{-\infty}^{\infty} d z^{\prime} w\left(z-z^{\prime}\right) \rho\left(z^{\prime}, s\right),
$$

yielding

$$
F(z, s)=e^{2} N \int_{-\infty}^{\infty} d z^{\prime} w\left(z-z^{\prime}\right) \rho\left(z^{\prime}, s\right) .
$$

To avoid confusion, notice that $w\left(z-z^{\prime}\right)$, here defined to denote an electric field per unit charge, is sometimes used to represent an electric potential per unit charge [22].

Alternatively, we can choose to work in the frequency domain using instead the impedance per unit length $\hat{Z}(k)$ [23] which is defined by $\tilde{E}_{s}(k)=-\hat{Z}(k) \tilde{I}(k)$, where $\tilde{E}_{s}(k)$ is the Fourier transform of the longitudinal field $E_{s}(z)$ due to a steady current $I(z)$, with $\tilde{I}(k)=-e N \beta c \tilde{\rho}(k)$ and

$$
\begin{gathered}
\tilde{E}_{s}(k)=\frac{1}{2 \pi} \int_{-\infty}^{\infty} d z e^{-i k z} E_{s}(z), \\
\tilde{\rho}(k)=\frac{1}{2 \pi} \int d z e^{-i k z} \rho(z) .
\end{gathered}
$$

By the inverse Fourier transformation of (4) we then have

$$
F(z, s)=-e E_{s}(z, s)=-e^{2} N \beta c \int_{-\infty}^{\infty} d k \hat{Z}(k) \tilde{\rho}(k, s) e^{i k z} .
$$

It follows from (4), (6), and (7) that

$$
w(z)=-\frac{c \beta}{2 \pi} \int_{-\infty}^{\infty} d k \hat{Z}(k) e^{i k z} .
$$

The minus sign on the RHS of the above equation follows from our convention that a positive $w$ corresponds to energy gain for a particle. Incidentally, notice that there may be models for the impedance in which the integral (8) does not exist, but where (7) may still be well defined thanks to the cutoff provided by $\tilde{\rho}(k, s)$. Models of impedance relevant for studying the microbunching instabilities are reviewed in the Appendix.

The Vlasov equation for the phase space density $f\left(x, p_{x}, z, \delta ; s\right)$ with Hamiltonian $H$ takes the form 


$$
\frac{\partial f}{\partial s}+p_{x} \frac{\partial f}{\partial x}+\left(\frac{\delta}{R}-x k_{x}\right) \frac{\partial f}{\partial p_{x}}-\frac{x}{R} \frac{\partial f}{\partial z}+\frac{F(z, s)}{p_{0}} \frac{\partial f}{\partial \delta}=0 .
$$

Next, we are interested in examining beams with vanishing transverse emittance. In order to derive a Vlasov equation governing such beams, it is useful to introduce a new set of coordinates describing the purely betatron part of the horizontal motion [24]:

$$
\begin{gathered}
x_{\beta}=x-\delta D, \\
p_{x \beta}=p_{x}-\delta D^{\prime}, \\
z_{\beta}=z+x D^{\prime}-p_{x} D, \\
\delta_{\beta}=\delta,
\end{gathered}
$$

where $D=D(s)$ is the dispersion function satisfying $D^{\prime \prime}+$ $k_{x} D=1 / R$. The transformation to the new coordinates has a generating function of the second kind

$$
F_{2}=p_{x \beta}\left(x-\delta_{\beta} D\right)+x \delta_{\beta} D^{\prime}+\left(z-\delta_{\beta} \frac{D D^{\prime}}{2}\right) \delta_{\beta},
$$

with $x_{\beta}=\partial F_{2} / \partial p_{x \beta}, p_{x}=\partial F_{2} / \partial x$, etc. This transformation yields the new Hamiltonian $H_{\beta}=H+\partial F_{2} / \partial s$,

$$
H_{\beta}=\frac{1}{2}\left(p_{x \beta}^{2}+k_{x} x_{\beta}^{2}\right)-\frac{D}{2 R} \delta_{\beta}^{2}-\frac{1}{p_{0}} \int_{-\infty}^{\zeta} F\left(z^{\prime}, s\right) d z^{\prime},
$$

where we set $\zeta=z_{\beta}-x_{\beta} D^{\prime}+p_{x \beta} D$. The resulting Vlasov equation is then

$$
\begin{gathered}
\frac{\partial f}{\partial s}+\left[p_{x \beta}-\frac{F(\zeta, s)}{p_{0}} D\right] \frac{\partial f}{\partial x_{\beta}}-\left[k_{x} x_{\beta}+\frac{F(\zeta, s)}{p_{0}} D^{\prime}\right] \frac{\partial f}{\partial p_{x \beta}} \\
-\delta_{\beta} \frac{D}{R} \frac{\partial f}{\partial z_{\beta}}+\frac{F(\zeta, s)}{p_{0}} \frac{\partial f}{\partial \delta_{\beta}}=0
\end{gathered}
$$

where we now regard $f$ as a function of the new variables.

The distribution of a beam with vanishing transverse emittance can be written as

$$
f=\hat{\delta}\left(x_{\beta}\right) \hat{\delta}\left(p_{x \beta}\right) f_{z}\left(z_{\beta}, \delta_{\beta}, s\right),
$$

where $\hat{\delta}$ is the Dirac delta function. In the presence of collective effects, a density function of the form (17) is not an exact solution of (16). In other words, a beam with initial vanishing horizontal emittance will see its emittance grow as a consequence of collective forces. We assume that such an emittance growth will be sufficiently small that (17) remains close to the actual solution. If this is the case, we can obtain a reduced 2D Vlasov equation for the longitudinal motion by inserting (17) into (16) and integrating over the transverse variables $x_{\beta}$ and $p_{x \beta}$ :

$$
\frac{\partial f_{z}}{\partial s}-\delta_{\beta} \frac{D}{R} \frac{\partial f_{z}}{\partial z_{\beta}}+\frac{F\left(z_{\beta}, s\right)}{p_{0}} \frac{\partial f_{z}}{\partial \delta_{\beta}}=0 .
$$

We might say that (17) satisfies (16) in the sense of an average over transverse phase space, if (18) is satisfied.

For brevity, for the rest of this and the next section we will drop the subscript $\beta$ in the notation for the coordinates and the subscript $z$ from the longitudinal density $f_{z}$. Also, we will find it convenient to regard $p$ in $\delta=\left(p-p_{0}\right) / p_{0}$ as the dynamical variable instead of $\delta$. In terms of the dynamical variables $(z, p)$, Eq. (18) can be rewritten as

$$
\frac{\partial f}{\partial s}-\frac{p-p_{0}}{p_{0}} \frac{D}{R} \frac{\partial f}{\partial z}+F(z, s) \frac{\partial f}{\partial p}=0 .
$$

For use in the next section, we report the mapping for a particle trajectory from $s$ to $s^{\prime}$ in the absence of collective effects. Using standard notation this can be written as $\mathcal{M}^{\text {ext }}:(z, p) \rightarrow\left(z^{\prime}, p^{\prime}\right)$ with

$$
z^{\prime}=z+\frac{p-p_{0}}{p_{0}}\left[R_{56}\left(s^{\prime}\right)-R_{56}(s)\right], \quad p^{\prime}=p,
$$

where $R_{56}(s)=-\int_{s_{0}}^{s} d \tau D(\tau) / R(\tau)$, having assumed that at the entry $s=s_{0}$ of the bunch compressor $R_{56}\left(s_{0}\right)=0$. Incidentally, notice that if $D=D^{\prime}=0$ at the entrance of a bend, and assuming that the transverse focusing is negligible, from $D^{\prime \prime}=1 / R$ it follows that $D(s)$ and $R(s)$ have the same sign so that $R_{56}(s)$ is negative over the first dipole of a chicane. In the next bend the sign of the radius of curvature $R$ changes and $R_{56}$ grows positive. A bunch compressor is designed so that $R_{56}$ remains positive through the last dipole of the chicane, see Fig. 6.

This concludes our discussion for particle motion in bunch compressors. As for longitudinal motion in an accelerating structure, we adopt the following model of the Vlasov equation:

$$
\frac{\partial f}{\partial s}+\left[\frac{\Delta E_{\mathrm{cav}}}{L_{\mathrm{cav}}} \sin \left(-\omega_{\mathrm{rf}} z / c+\phi_{s}\right)+F(z, s)\right] \frac{\partial f}{\partial p}=0,
$$

where $\Delta E_{\text {cav }}$ is the energy gain by a synchronous particle through a cavity of length $L_{\text {cav }} ; \omega_{\text {rf }}$ and $\phi_{s}$ are the cavity frequency and phase. According to this model, in the absence of collective effects the mapping for a particle trajectory from $s$ to $s^{\prime}$ reads $\mathcal{M}^{\text {ext }}:(z, p) \rightarrow\left(z^{\prime}, p^{\prime}\right)$ with

$$
z^{\prime}=z, p^{\prime}=p+\left(s^{\prime}-s\right) \frac{\Delta E_{\mathrm{cav}}}{L_{\mathrm{cav}}} \sin \left(-\omega_{\mathrm{rf}} z / c+\phi_{s}\right) .
$$

\section{SOLVING THE VLASOV EQUATION}

The main purpose of this paper is to indicate an efficient method to solve the Vlasov equations (19) and (21).

A more direct way of stating the local beam density conservation along the particle trajectories expressed by a 
Vlasov equation is to write $f\left(z^{\prime}, s^{\prime}\right)=f(z, s)$, where $z^{\prime}=$ $\mathcal{M}_{s \rightarrow s^{\prime}} z$ is the volume preserving map describing particle trajectories from "time" $s$ to $s^{\prime}$. More suggestively the same equation can be restated as

$$
f\left(z^{\prime}, s^{\prime}\right)=f\left(\mathcal{M}_{s \rightarrow s^{\prime}}^{-1}\left(z^{\prime}\right), s\right) .
$$

This form of the Vlasov equation is appealing in that it suggests a straightforward and effective method of solution in which a discretized version of (23) is applied at each time step using an approximation for the map $\mathcal{M}_{s \rightarrow s^{\prime}}$. This method is widely used and known in the literature under a variety of names: method of characteristics, semiLagrangian, Perron-Frobenius, etc. Typically $f$ is represented on a static, uniform Cartesian grid with polynomial or other interpolation schemes at off grid points. To solve Eq. (19) and (21), we would like to keep the convenience of using a uniform Cartesian grid. However, because a strong energy/position correlation is applied to the beam to achieve the desired compression, this would be quite inefficient - the support of the beam density function $f$ is non-negligible only on a small portion of a full Cartesian grid, as seen in the top picture of Fig. 1. We sidestep this difficulty by introducing a new coordinate system in which the above-mentioned correlation has been removed.

To this end, we need a suitable definition of beam correlation. Let us define the correlation or "chirp" function at the entrance $s=s_{0}$ of a chicane in terms of the known beam density $f_{0}\left(z, p ; s_{0}\right)$ at $s_{0}$ :

$$
\alpha\left(z, s_{0}\right)=\int_{-\infty}^{\infty} f_{0}\left(z, p, s_{0}\right) p d p / \int_{-\infty}^{\infty} f_{0}\left(z, p, s_{0}\right) d p .
$$

We then define a rule to propagate the correlation function for $s>s_{0}$ in terms of the dynamics unperturbed by collective effects.

In a bunch compressor, given $\alpha(z, s)$ at $s$ as a function of $z$, the value at $z^{\prime}$ of $\alpha\left(z^{\prime}, s^{\prime}\right)$ at a later time $s^{\prime}>s$ is

$$
\alpha\left(z^{\prime}, s^{\prime}\right)=\alpha(z, s),
$$

where $z$ in the RHS is determined for a given $z^{\prime}$ as a solution of the algebraic equation,

$$
z^{\prime}=z+\frac{\alpha(z, s)-p_{0}}{p_{0}}\left[R_{56}\left(s^{\prime}\right)-R_{56}(s)\right]
$$

We make the assumption that during its evolution $\alpha(z, s)$ remains a single-valued function in $z$. A sketch of the chirp-function propagation is shown in the top picture of Fig. 2.

In an accelerating structure, we let the correlation function evolve according to

$$
\alpha\left(z^{\prime}, s^{\prime}\right)=\alpha(z, s)+\left(s^{\prime}-s\right) \frac{\Delta E_{\mathrm{cav}}}{L_{\mathrm{cav}}} \sin \left(-\omega_{\mathrm{rf}} z / c+\phi_{s}\right),
$$

with $z^{\prime}=z$.
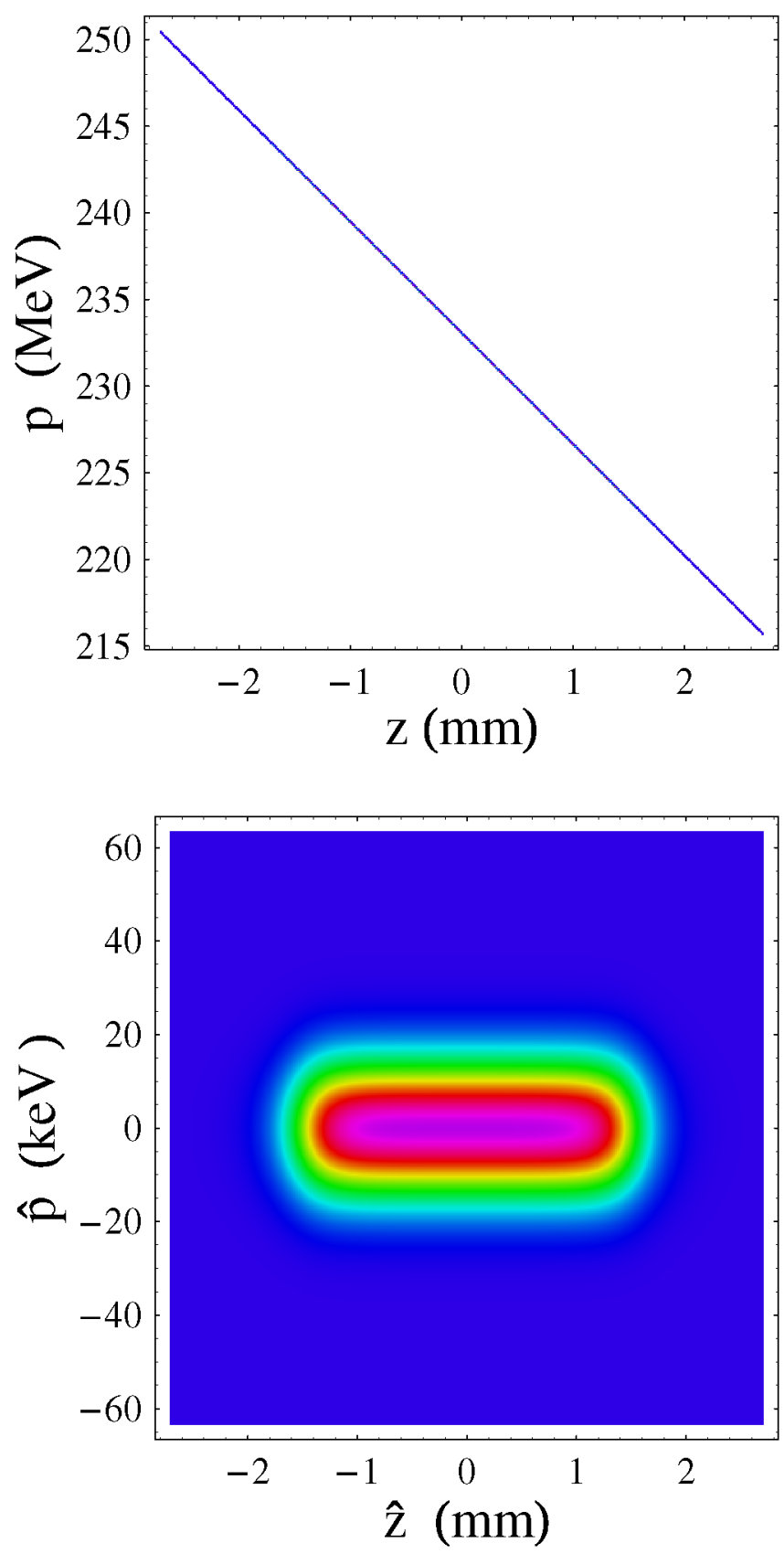

FIG. 1. (Color) Support of the beam density function in phase space at the entrance of a bunch compressor in the $(z, p)$ coordinates (top picture). The beam density of the same beam is plotted in the $(\hat{z}, \hat{p})$ coordinates (bottom picture) where the beam linear chirp has been subtracted. The phase space in the bottom picture can be more efficiently gridded by a Cartesian mesh.

The meaning of this rule is clear: in the absence of collective forces, it implies that the correlation function $\alpha(z, s)$ at $s>s_{0}$ still satisfies (24) with $f_{0}$ replaced by the beam density $f$ at $s$. With this definition $\alpha(z, s)$ becomes a known function which we can use to define the new dynamical variables $(\hat{z}, \hat{p})$ by the transformation 

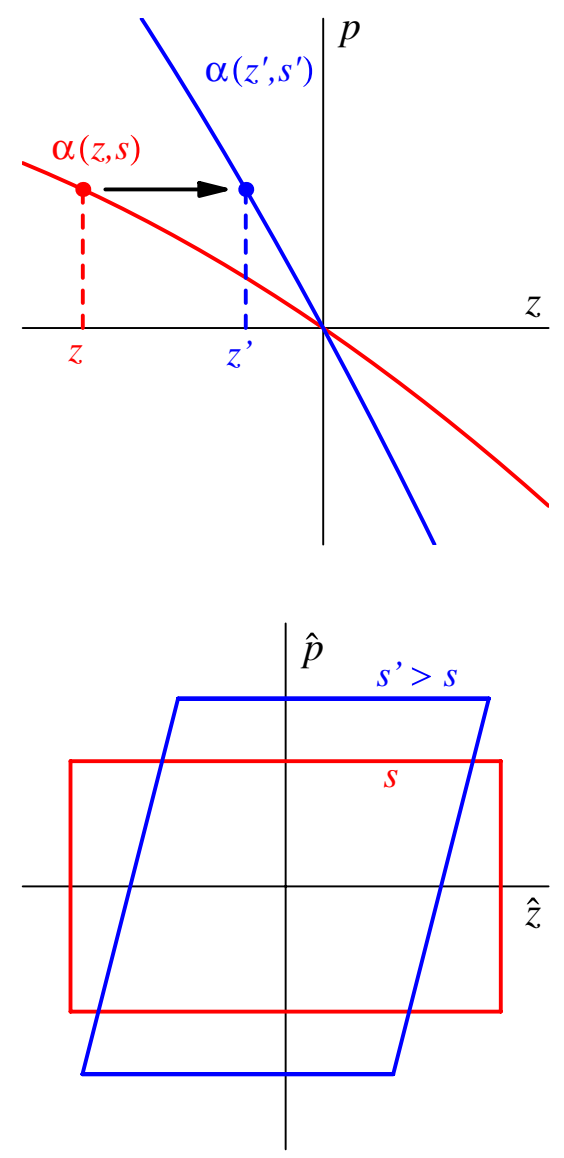

FIG. 2. (Color) Schematic for the evolution of the chirp function in a bunch compressor (top figure) and dynamics in the $(\hat{z}, \hat{p})$ phase space for a beam with linear chirp (bottom figure).

$$
\begin{gathered}
\mathcal{A}:(z, p) \rightarrow(\hat{z}, \hat{p}) \quad \\
\hat{z}=z, \\
\hat{p}=p-\alpha(z, s) .
\end{gathered}
$$

As desired, the support of the beam distribution in the new variables is better matched to a Cartesian grid, as in the bottom picture of Fig. 1.

Propagation of the density function in (23) requires a suitable approximation of the mapping for the particle trajectory $\hat{\mathcal{M}}_{s \rightarrow s+\Delta s}$ over the time step $\Delta s$. The ^denotes that the map is in terms of the new variables. A convenient choice is given by the second order integrator

$$
\hat{\mathcal{M}}_{s \rightarrow s+\Delta s} \simeq \hat{\mathcal{M}}_{\Delta s / 2}^{c} \hat{\mathcal{M}}_{\Delta s}^{\mathrm{ext}} \hat{\mathcal{M}}_{\Delta s / 2}^{c},
$$

where $\hat{\mathcal{M}}_{\Delta s}^{\text {ext }}$ represents the mapping under the action of the external forces from $s$ to $s^{\prime}=s+\Delta s$ and $\hat{\mathcal{M}}_{\Delta s / 2}^{c}$ yields a half-kick under the collective force. These elementary mappings in the new coordinates can be recovered from the corresponding mappings in the original coordinates by means of the transformation $\mathcal{A}: \hat{\mathcal{M}}^{i}=\mathcal{A} \mathcal{M}^{i} \mathcal{A}^{-1}$, where $i=$ " $c$ " or "ext." The maps $\mathcal{M}^{\text {ext }}$ in a bunch compressor and accelerating structure were reported in (20) and (22); $\mathcal{M}^{c}$ is simply $z^{\prime}=z$ and $p^{\prime}=p+$ $\Delta s F(z, s)$, with the collective-force $F(z, s)$ calculated using the current beam density.

The form of the collective-force half-kick is formally the same as in the original coordinates, $\hat{\mathcal{M}}_{\Delta s / 2}^{c}:(\hat{z}, \hat{p}) \rightarrow$ $\left(\hat{z}^{\prime}, \hat{p}^{\prime}\right)$ with

$$
\begin{gathered}
\hat{p}^{\prime}=\hat{p}+(\Delta s / 2) F(\hat{z}), \\
\hat{z}^{\prime}=\hat{z} .
\end{gathered}
$$

In a bunch compressor the mapping under the external forces in the new variables is more involved but still easy to write, $\hat{\mathcal{M}}^{\text {ext }}:(\hat{z}, \hat{p}) \rightarrow\left(\hat{z}^{\prime}, \hat{p}^{\prime}\right)$ with

$$
\begin{gathered}
\hat{z}^{\prime}=z^{\prime}=z+\left(p-p_{0}\right) d \bar{R}_{56} \\
=\hat{z}+\left[\hat{p}+\alpha(\hat{z}, s)-p_{0}\right] d \bar{R}_{56}, \\
\hat{p}^{\prime}=p^{\prime}-\alpha\left(z^{\prime}, s^{\prime}\right)=p-\alpha\left(z+\left(p-p_{0}\right) d \bar{R}_{56}, s^{\prime}\right) \\
=\hat{p}+\alpha(\hat{z}, s)-\alpha\left(\hat{z}+\left[\hat{p}+\alpha(\hat{z}, s)-p_{0}\right] d \bar{R}_{56}, s^{\prime}\right),
\end{gathered}
$$

where for brevity we have denoted $d \bar{R}_{56}=d \bar{R}_{56}\left(s, s^{\prime}\right)=$ $\left[R_{56}\left(s^{\prime}\right)-R_{56}(s)\right] / p_{0}$. The inverse of the above map, which in view of (23) is of more direct interest, reads

$$
\begin{gathered}
\hat{z}=\hat{z}^{\prime}-\left[\hat{p}^{\prime}+\alpha\left(\hat{z}^{\prime}, s\right)-p_{0}\right] d \bar{R}_{56} \\
\hat{p}=\hat{p}^{\prime}+\alpha\left(\hat{z}^{\prime}, s^{\prime}\right)-\alpha\left(\hat{z}^{\prime}+\left[\hat{p}^{\prime}+\alpha\left(\hat{z}^{\prime}, s\right)-p_{0}\right] d \bar{R}_{56}, s\right) .
\end{gathered}
$$

To get a better grasp of this mapping, consider the simple case of a beam with a linear chirp: i.e.

$$
\alpha(z, s)=\alpha_{1}(s) z+p_{0} .
$$

We can determine the chirp function at a later $s^{\prime}>s$ by solving Eqs. (25) and (26). We find that the chirp function at $s^{\prime}$ is still a linear function of $z: \alpha\left(z^{\prime}, s^{\prime}\right)=\alpha_{1}\left(s^{\prime}\right) z^{\prime}+p_{0}$ with

$$
\alpha_{1}\left(s^{\prime}\right)=\frac{\alpha_{1}(s)}{1+\alpha_{1}(s) d \bar{R}_{56}\left(s, s^{\prime}\right)}
$$

By using Eqs. (33) and (34) we find

$$
\begin{gathered}
\hat{z}^{\prime}=\hat{z}\left[1+\alpha_{1}(s) d \bar{R}_{56}\left(s, s^{\prime}\right)\right]+\hat{p} d \bar{R}_{56}\left(s, s^{\prime}\right), \\
\hat{p}^{\prime}=\frac{\hat{p}}{1+\alpha_{1}(s) d \bar{R}_{56}\left(s, s^{\prime}\right)} .
\end{gathered}
$$

Observe that, unlike the canonical momentum $p$, the coordinate $\hat{p}$ expressing the uncorrelated energy spread is not invariant. In particular, the $\hat{p}$-direction stretches [in a bunch compressor the number $1+\alpha_{1}(s) d \bar{R}_{56}\left(s, s^{\prime}\right)$ is generally smaller than unity] while $\hat{z}$ is compressed. Moreover, as indicated in the last term in (39), the degree of com- 
pression on $\hat{z}$ depends, albeit weekly, on $\hat{p}$. A graphical illustration of the action of the linear transformation (39) and (40) is presented in the bottom picture of Fig. 2.

Finally, in an accelerating structure the mapping $\hat{\mathcal{M}}^{\text {ext }}:(\hat{z}, \hat{p}) \rightarrow\left(\hat{z}^{\prime}, \hat{p}^{\prime}\right)$ is simply the identity $\hat{p}^{\prime}=\hat{p}$ and $\hat{z}^{\prime}=\hat{z}$.

\section{ALGORITHM}

We represent the density function in the variables $(\hat{z}, \hat{p})$ on a Cartesian grid with a fixed number of $\left(2 N_{\hat{z}}+1\right) \times$ $\left(2 N_{\hat{p}}+1\right)$ nodes but with varying cell sizes $\Delta_{\hat{z}}^{k}$ and $\Delta_{\hat{p}}^{k}$ the index $k$ refers to the time step). Grid adaptation follows the support of the beam density function in phase space as the beam contracts in position $\hat{z}$ and expands in the canonical momentum $\hat{p}$. Following the time-splitting method outlined in the previous section, we first advance the density function by $\Delta s$ under the mapping $\hat{\mathcal{M}}_{\Delta s}^{\text {ext }}$ for the unperturbed lattice defined in (33) and (34), and then advance the density function under the collective-force kick $\hat{\mathcal{M}}_{\Delta s / 2}^{c}$ defined in (31) and (32). The mappings for two adjacent half kicks can, of course, be lumped together into a single $\Delta s$ collective kick.

Suppose that at time $s=k \Delta s$ the density function $f_{i j}=$ $f\left(\hat{z}_{i}, \hat{p}_{j}\right)$ is known on the grid nodes $\hat{z}_{i}, \hat{p}_{j}$ specified as $\hat{z}_{i}=$ $i \Delta_{\hat{z}}^{k}$, with $i=-N_{\hat{z}},-N_{\hat{z}}+1, \ldots, N_{\hat{z}}$ and $\hat{p}_{j}=j \Delta_{\hat{p}}^{k}$ with $j=-N_{\hat{p}},-N_{\hat{p}}+1, \ldots, N_{\hat{p}}$. Assume that the chirp function $\alpha\left(\hat{z}_{i}, s\right)$ on the grid $\hat{z}_{i}$ is also known.

To propagate the density from previous time $s=k \Delta s$ to current time $s^{\prime}=s+\Delta s=(k+1) \Delta s$ we undertake the following steps [25].

(i) Adapt the new grid sizes according to

$$
\begin{gathered}
\Delta_{\hat{z}}^{k+1}=\Delta_{\hat{z}}^{k} / C, \\
\Delta_{\hat{p}}^{k+1}=\Delta_{\hat{p}}^{k} C,
\end{gathered}
$$

where the compression factor $C=\left[1+d R_{56}\left(s, s^{\prime}\right) \times\right.$ $\left.\left.\frac{d \alpha(\hat{z}, s)}{d \hat{z}}\right|_{\hat{z}=0}\right]^{-1}$ depends on the slope of the chirp function $\alpha(\hat{z}, s)$ at $\hat{z}=0$. The slope is calculated from the coefficients of the spline interpolation of $\alpha\left(\hat{z}_{i}, s\right)$.

(ii) Advance the chirp function from $s$ to current time $s^{\prime}$ according to (25) and (26). If (25) and (26) apply, first find the images of the grid points $\hat{z}_{i}$ under the map (26); these images in general will not fall on grid points of the grid at current time $s^{\prime}$ but can be used to create a cubic spline representation for $\alpha$ at $s^{\prime}$. The chirp function is then evaluated on the grid points of the current grid at $s^{\prime}$ by spline interpolation.

(iii) Determine the backward images of the nodes $\left(\hat{z}_{i}^{\prime}=\right.$ $i \Delta_{\hat{z}}^{k+1}, \hat{p}_{j}^{\prime}=j \Delta_{\hat{p}}^{k+1}$ ) under the inverse of the map $\hat{\mathcal{M}}^{\text {ext }}$ defined in (35) and (36):

$$
\hat{z}=\hat{z}_{i}^{\prime}-\left[\hat{p}_{j}^{\prime}+\alpha\left(\hat{z}_{i}^{\prime}, s\right)-p_{0}\right] d \bar{R}_{56},
$$

$\hat{p}=\hat{p}_{j}^{\prime}+\alpha\left(\hat{z}_{i}^{\prime}, s^{\prime}\right)-\alpha\left(\hat{z}_{i}^{\prime}+\left[\hat{p}_{j}^{\prime}+\alpha\left(\hat{z}_{i}^{\prime}, s\right)-p_{0}\right] d \bar{R}_{56}, s\right)$.

Notice that to evaluate this mapping we make use of the chirp function $\alpha$ both at present $s^{\prime}$ and previous time $s$. In (44) we use spline interpolation when the argument of $\alpha$ falls between grid points.

(iv) In the grid at $s$ identify the nodes neighboring the point $(\hat{z}, \hat{p})$ calculated in (43) and (44). Use the values of the density function at time $s$ on these nodes to determine $f(\hat{z}, \hat{p} ; s)$ by interpolation. We found a local 16-point interpolation scheme using bicubic polynomials quite effective. Identify the value $f\left(\hat{z}_{i}^{\prime}, \hat{p}_{j}^{\prime}, s^{\prime}\right)$ of the density function at current time $s^{\prime}$ on the node $\left(\hat{z}_{i}^{\prime}, \hat{p}_{j}^{\prime}\right)$ as $f\left(\hat{z}_{i}^{\prime}, \hat{p}_{j}^{\prime}, s^{\prime}\right)=$ $f(\hat{z}, \hat{p}, s)$.

(v) Integrate the phase density $f\left(\hat{z}_{i}^{\prime}, \hat{p}_{j}^{\prime}, s^{\prime}\right)$ with respect to canonical momentum to obtain the longitudinal charge density.

(vi) Calculate the Fourier transform of the charge density and combine it with the impedance to determine the collective force and the corresponding kick at $\hat{z}_{i}^{\prime}$.

(vii) Advance the beam density function under the collective-force kick. Because the kick depends only on the position (not the canonical momentum) coordinate, only 1D interpolations are necessary to determine the density function [6]. This interpolation is done by cubic splines.

Evaluation of the Fourier transform of the charge density in item (vi) and determination of the collective force is done by a fast-Fourier-transform algorithm (FFT) along the lines of Ref. [11].

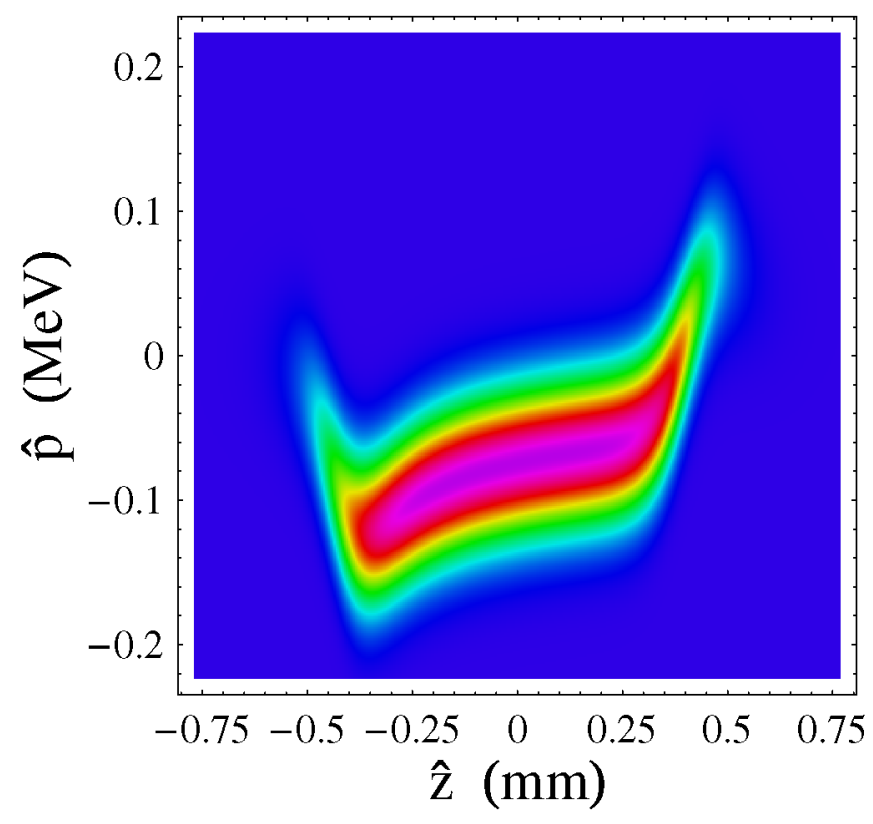

FIG. 3. (Color) Beam density function in the $(\hat{z}, \hat{p})$ phase space at the exit of a bunch compressor and in the presence of CSR. The head of the bunch is at $\hat{z}>0$. 
An example of numerical solution obtained using this integration method is shown in Fig. 3. This is a snapshot of the beam density at the end of the first bunch compressor of the free electron laser radiation for multidisciplinarity investigations (FERMI) linac [26] starting from the beam density shown in Fig. 2. The only collective effect present here is coherent synchrotron radiation (CSR).

\section{VALIDATION OF ALGORITHM AND CODING}

We ran a number of tests to check the validity of the algorithm and its implementation in a numerical code. We typically considered a model of "smooth" flattop beam with Gaussian energy spread $f(z, p)=h\left(z, p-p_{0}-\right.$ $\left.\alpha_{1} z\right)$, where $h(z, p)=\rho_{\mathrm{flt}}(z) \exp \left(-p^{2} / 2 \sigma_{p}^{2}\right) / \sqrt{2} \pi \sigma_{p}$ and $\rho_{\mathrm{flt}}(z)=\left\{\tanh \left[\left(z+l_{b}\right) / f_{r}\right]-\tanh \left[\left(z-l_{b}\right) / f_{r}\right]\right\} / 4 l_{b}$. In the above equation $2 l_{b}$ is the bunch length and $f_{r}$ a parameter controlling the roll-off of the density profile at the beam ends. We also added a sinusoidal perturbation to the charge density $\rho(z)=\left[1+A_{i} \sin (k z)\right] \rho_{\mathrm{flt}}(z)$ with small amplitude perturbation $A_{i}$.

In the numerical examples shown in this section, we adopted the parameters for the first bunch compressor of the FERMI FEL complex [26], consisting of four bends of $0.5 \mathrm{~m}$ length and $R=7.38 \mathrm{~m}$ radius of curvature. The electron beam passes the bunch compressor with energy $p_{0}=233 \mathrm{MeV}$. We considered a beam with uncorrelated energy spread $\sigma_{p}=10 \mathrm{keV}$, corresponding to $\sigma_{\delta}=$ $\sigma_{p} / p_{0}=4.3 \times 10^{-5}$, and linear chirp $\alpha(z)=\alpha_{1}\left(s_{0}\right) z+$ $p_{0}$. At the entrance of the first bunch compressor $\alpha_{1}\left(s_{0}\right) / p_{0}=-27.5 \mathrm{~m}^{-1}$, resulting into a 3.5 compression factor, and $l_{b}$ is about $l_{b}=1.5 \mathrm{~mm}$. A typical mesh size (as in Figs. 1 and 3) is $400 \times 400$.

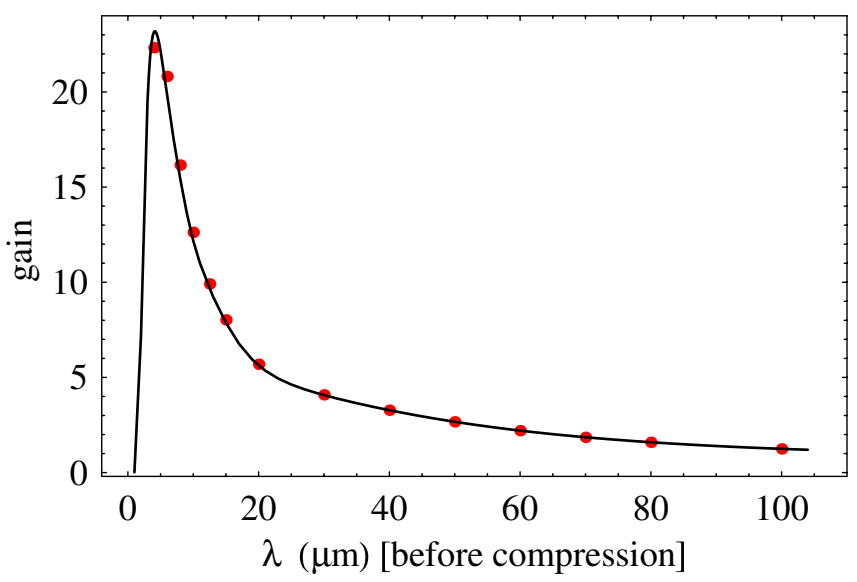

FIG. 4. (Color) Gain function through the FERMI first bunch compressor at finite current, as determined from numerical solutions of the Vlasov equation (dots) and linear theory (solid line). The peak current before compression is $I=95.5 \mathrm{~A}(I=$ 336.2 A after compression). CSR is the only collective effect included in the calculation.

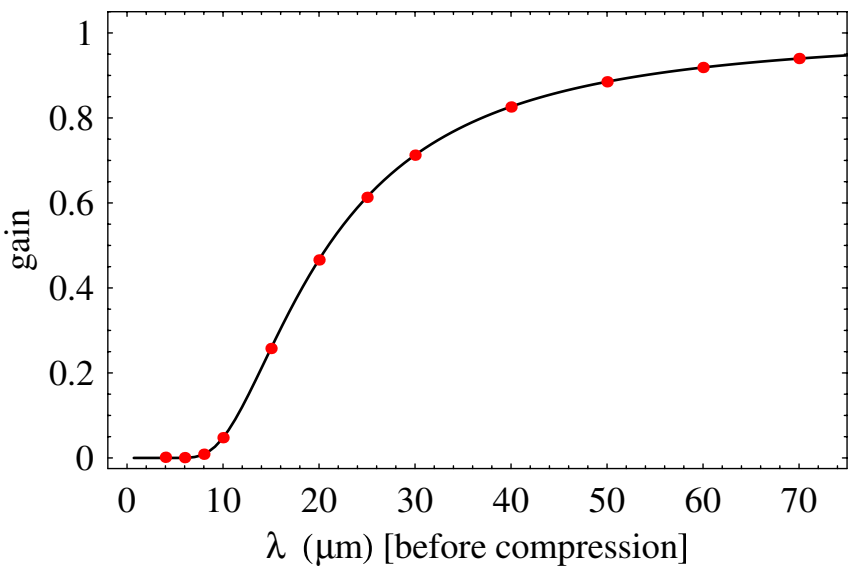

FIG. 5. (Color) The exact (solid line) gain function through the FERMI first bunch compressor at vanishing current is compared to that determined from numerical solutions of the Vlasov equation (dots).

In one test we compared the beam density propagated to the end of the bunch compressor in the absence of collective effects with the exact solution (which is easy to determine because the dynamics is linear). This helped us decide in favor of a 16-point bicubic interpolation scheme rather than a less efficient 4-point interpolation. In other tests we turned on the collective effects and specifically CSR to make contact with the linear theory by looking at the amplification of initial small sinusoidal perturbations. If we denote with $\rho_{i}$ and $\rho_{f}$ the peak longitudinal beam densities at the start and end of the bunch compressor [27] the gain function for the given wavelength is conventionally defined as $g=\left(A_{f} / \rho_{f}\right) /\left(A_{i} / \rho_{i}\right)$, where $A_{i}$ and $A_{f}$ are the initial and final amplitude of the chargedensity perturbation at wavelength $\lambda$. The good agreement with the theory [2] as shown in Fig. 4 was achieved with a choice of the integration step $\Delta s$ of about $5 \%$ of the dipoles length. Finally, in Fig. 5 we report a comparison between numerical calculation and the analytical formula [3],

$$
g(k, s)=\exp \left[-\frac{1}{2} k^{2} \sigma_{\delta}^{2} R_{56}^{2}(s) C(s)\right],
$$

for the gain function in the absence of collective effects. In the equation above, $C(s)=1 /\left[1+\alpha_{1}(s) R_{56}(s) / p_{0}\right]$ is the compression factor at $s$ for a beam with linear chirp.

\section{APPROXIMATE ACCOUNT OF TRANSVERSE MOTION EFFECTS ON LONGITUDINAL DYNAMICS}

The most important limitation to a purely 2D model of dynamics is neglect of a smearing effect on microbunching caused in a bunch compressor by a finite horizontal emittance, which may result in substantially smaller gain curves for the microbunching instability [1-3]. While a fully accurate account requires solving the equations of motion in $4 \mathrm{D}$, in this section we propose a heuristic model 
that appears to yield some acceptable degree of approximation when compared to linear theory. We suppose, without strong justification, that a solution of the 4D Vlasov equation expressed in terms of the "beta" coordinates of Sec. II, maintains an approximate factorization between longitudinal and transverse motion:

$$
f \approx g\left(x_{\beta}, p_{x \beta}\right) f_{z \beta}\left(z_{\beta}, \delta_{\beta}\right) .
$$

For the horizontal distribution, we assume a Gaussian density matched to the lattice,

$$
g\left(x_{\beta}, p_{x \beta}\right)=\frac{1}{2 \pi \varepsilon_{x}} \exp \left(-\frac{I_{\beta}\left(x_{\beta}, p_{x \beta}\right)}{2 \varepsilon_{x}}\right),
$$

where $I_{\beta}\left(x_{\beta}, p_{x \beta}\right)=\gamma_{x} x_{\beta}^{2}+2 \alpha_{x} x_{\beta} p_{x \beta}+\beta_{x} p_{x \beta}^{2}$ is the familiar Courant invariant. Notice that the factorization (46) would be exactly preserved in the absence of collective effects.

Next, insert the ansatz (46) into the 4D Vlasov equation (16) and find

$$
g \frac{\partial f_{z \beta}}{\partial s}-\delta_{\beta} \frac{D}{R} g \frac{\partial f_{z \beta}}{\partial z_{\beta}}+F(\zeta, s) g \frac{\partial f_{z \beta}}{\partial \delta_{\beta}}+T_{\beta}=0,
$$

where as in Sec. II, $\zeta$ is shorthand for $\zeta=z_{\beta}-x_{\beta} D^{\prime}+$ $p_{x \beta} D$ and

$$
\begin{aligned}
F(\zeta, s)= & e^{2} N \int_{-\infty}^{\infty} d z^{\prime} w\left(\zeta-z^{\prime}\right) \\
& \times \int d x d p_{x} d \delta f\left(x, p_{x}, z^{\prime}, \delta, s\right) \\
= & e^{2} N \int_{-\infty}^{\infty} d z^{\prime} w\left(\zeta-z^{\prime}\right) \\
& \times \int d x d p_{x} d \delta g\left(x-\delta D, p_{x}-\delta D^{\prime}\right) \\
& \times f_{z \beta}\left(z^{\prime}+x D^{\prime}-p_{x} D, \delta, s\right)
\end{aligned}
$$

with

$$
T_{\beta}=-F(\zeta) f_{z \beta}\left[D \frac{\partial g}{\partial x_{\beta}}+D^{\prime} \frac{\partial g}{\partial p_{x \beta}}\right] .
$$

In writing (48), we exploited the invariance of $I_{\beta}$ under the unperturbed dynamics: $d I_{\beta} / d s=0$. Finally, we remove the dependence of (48) on the transverse coordinates by averaging and obtain

$$
\frac{\partial f_{z \beta}}{\partial s}-\delta_{\beta} \frac{D}{R} \frac{\partial f_{z \beta}}{\partial z_{\beta}}+\frac{\partial f_{z \beta}}{\partial \delta_{\beta}} \int d x_{\beta} d p_{x \beta} F(\zeta, s) g\left(x_{\beta}, p_{x \beta}\right)=0 .
$$

In (50) we made use of the fact that the average of $T_{\beta}$ over the transverse phase space vanishes, as can be seen after integration by parts. In Eq. (50) the term

$$
F_{\mathrm{sm}}\left(z_{\beta}\right)=\int d x_{\beta} d p_{x \beta} F\left(z_{\beta}-x_{\beta} D^{\prime}+p_{x \beta} D\right) g\left(x_{\beta}, p_{x \beta}\right)
$$

is an effective longitudinal collective force accounting for the smearing from the horizontal emittance.

Upon carrying out some judicious changes of integration variables, one can verify that (51) reduces to

$$
F_{\mathrm{sm}}(z, s)=e^{2} N \int_{-\infty}^{\infty} d z^{\prime} w\left(z-z^{\prime}\right) \rho_{\mathrm{sm}}\left(z^{\prime}, s\right)
$$

where the smeared longitudinal density $\rho_{\mathrm{sm}}(z, s)$ is given by

$$
\rho_{\mathrm{sm}}(z, s)=\int_{-\infty}^{\infty} d t \frac{\exp \left(-t^{2} / 2 \sigma_{\perp}^{2}\right)}{\sqrt{2 \pi} \sigma_{\perp}} \rho(z+t, s),
$$

with the amplitude of the smearing $\sigma_{\perp}=\sqrt{2 \varepsilon_{x} \mathcal{H}}$ depending on the transverse lattice through the "curly $\mathrm{H}$ " function $\mathcal{H}=\gamma_{x} D^{2}+2 \alpha_{x} D D^{\prime}+\beta_{x}\left(D^{\prime}\right)^{2}$. In the frequency domain, the smearing has the form of a low-pass filter

$$
F_{\mathrm{sm}}(z, s)=-e^{2} N c \int_{-\infty}^{\infty} d k \hat{Z}(k) \tilde{\rho}(k, s) e^{i k z} e^{-k^{2} \sigma_{\perp}^{2} / 2},
$$

with a cutoff wavelength $\lambda_{c}$ defined by $k^{2} \sigma_{\perp}^{2} / 2 \simeq 1$ yielding $\lambda_{c}=2 \pi \sqrt{\varepsilon_{x} \mathcal{H}}$. A plot of $\mathcal{H}$ in the FERMI first bunch compressor is shown in Fig. 6.

That the smearing induced by a finite transverse emittance is related to the $\mathcal{H}(s)$ function is not surprising but it appears to have escaped notice. For example, after some simple manipulations involving the transfer matrix entries $R_{51}$ and $R_{52}$ the expression for the gain function in the absence of collective effects reported in [3] can be cast in the form

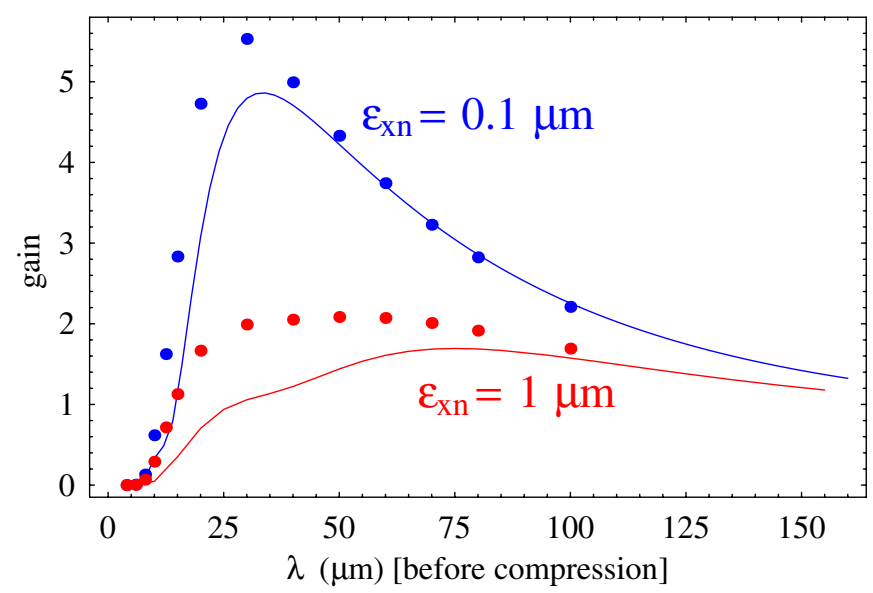

FIG. 6. (Color) $R_{56}(s)$ and $\mathcal{H}(s)$ functions in FERMI first bunch compressor. 

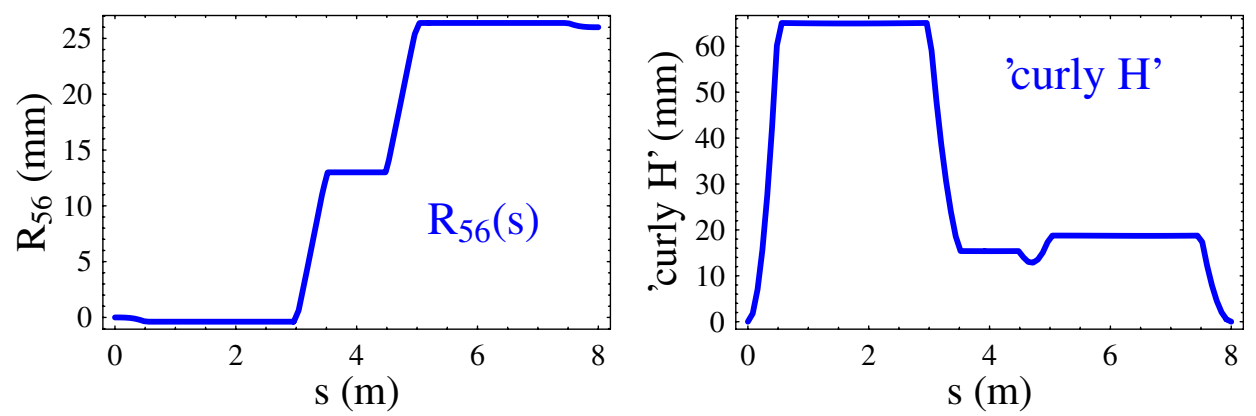

FIG. 7. (Color) Comparison between model of smearing from horizontal emittance discussed in this section (dots) and linear theory (lines). The gain curves are for the FERMI first bunch compressor and are shown for two values of normalized horizontal emittance. The beam peak current (before compression) is $I=191.1 \mathrm{~A}$ (twice the design value). The beam energy is $E=233 \mathrm{MeV}$, the energy spread $\sigma_{p}=10 \mathrm{keV}$.

$$
g(k, s)=\exp \left(-\frac{k^{2} C(s)}{2}\left[\sigma_{\delta}^{2} R_{56}^{2}(s)+\varepsilon_{x} \mathcal{H}(s)\right]\right)
$$

where $C(s)$ is the compression factor defined at the end of $\mathrm{V}$.

To test our model against linear theory [2], we evaluated the gain function through the FERMI first bunch compressor $\mathrm{BC} 1$ in the presence of CSR only (i.e. space-charge was not included) for a beam current $I=191$ A (before compression); this value is twice the FERMI design specification. We considered two values of horizontal emittance. The results are reported in Fig. 7 as dots. The solid lines are from linear theory. We observe some discrepancy at larger emittance with the agreement becoming better at smaller emittance.

In a second set of calculations shown here, we included the $\mathrm{L} 1$ section of the FERMI linac preceding $\mathrm{BC} 1$ and turned on space-charge in L1 (but CSR was the only collective effect present in the bunch compressor). At the entrance of the L1 section, the beam is at an energy of about $E=96 \mathrm{MeV}$ and is accelerated to $E=233 \mathrm{MeV}$ as it enters $\mathrm{BC} 1$ after traveling through five radio frequency (RF) structures. The length of L1 is about $36 \mathrm{~m}$. Spacecharge kicks as determined using impedance (A1) were applied once at the end of each drift and quadrupole, and

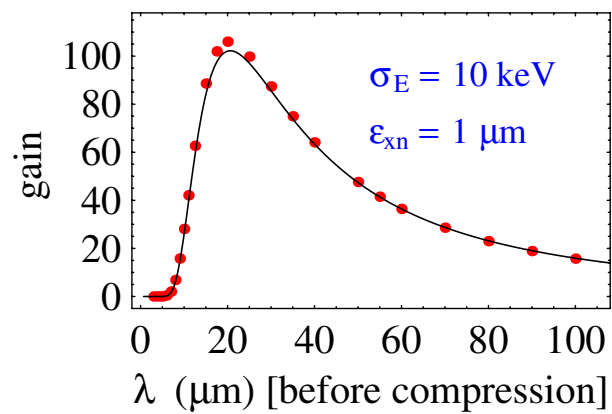

10 times within each cavity. The additional number of kicks applied in the cavities is required by the strong dependence of space-charge on beam energy.

In the calculation we defined the $r_{b}$ parameter [see (A1)] as $r_{b}=1.3\left[\left(\sigma_{x}^{2}+\sigma_{y}^{2}\right) / 2\right]^{1 / 2}$, where $\sigma_{x}^{2}=\beta_{x} \varepsilon_{x}, \sigma_{y}^{2}=$ $\beta_{y} \varepsilon_{y}$ are the local values of the transverse rms beam sizes (where we assumed $\gamma \varepsilon_{x}=\gamma \varepsilon_{y}=1 \mu \mathrm{m}$ ). The factor 1.3 is an attempt [28] to adjust the space-charge model to account for the actual transverse density distribution of the physical beam, which is closer to Gaussian than uniform (recall that the impedance (A1) presupposes a beam with uniformly transverse density and circular cross-section).

The calculation was done for a peak current $I=95.5 \mathrm{~A}$ (before compression). The gain functions through $\mathrm{L} 1+$ $\mathrm{BC} 1$ are shown in the two pictures in Fig. 8 as red dots and contrasted with the gain curves from linear theory (solid lines) for two values of the normalized horizontal emittance.

In the linear theory calculation, we used the same model of space-charge with the beam radius parameter $r_{b}$ set to $r_{b}=0.28 \mathrm{~mm}$, a value resulting the average of the $r_{b}$ defined above over the length of the linac L1. The energy was assumed to increase linearly over L1.

By comparing the two pictures in Fig. 8 we can appreciate the considerable impact that a horizontal emittance

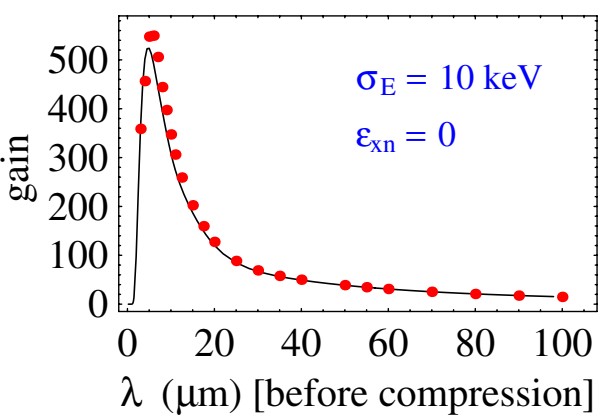

FIG. 8. (Color) Gain curves through FERMI L1 and BC1 as determined from linear theory (solid line) and numerical solutions of the Vlasov equation, with nonvanishing (left picture) and vanishing (right picture) transverse emittance. Space-charge was included in L1 but not in $\mathrm{BC} 1$ and calculated based on the beam sizes resulting from $\gamma \varepsilon_{x}=\gamma \varepsilon_{y}=1 \mu \mathrm{m}$ in both pictures. CSR was included in BC1. 
$\varepsilon_{x n}=1 \mu \mathrm{m}$ has on softening the gain function. The agreement with linear theory is satisfactory but, we should emphasize, this is for a case where the overwhelming contribution to the gain function results from space-charge in the linac trailing the bunch compressor.

\section{DISCUSSION AND CONCLUSIONS}

The direct Vlasov methods discussed in this paper are ideally suited to study short-scale phenomena, like the microbunching instability, that are more difficult to access using other simulation techniques. A systematic application of our solver to the study of microbunching in the overall FERMI linac, as well as a close comparison with macroparticle simulations and a more thorough assessment of the applicability of the model presented in Sec. VI, is currently underway and will be reported elsewhere.

We end by pointing to a limitation of our Vlasov solver in its current form. Beam features of a scale larger than that involved with microbunching (including large energy spread developing at the ends of flattop beams or curvatures of the beam densities due to the RF wave nonlinearities and RF structure wakefields) may be handled but not in full generality by the algorithm presented here.

A difficulty would arise if collective forces substantially affected the beam position/energy correlation in the longitudinal phase space. This would make it necessary to redefine the rule for the evolution of the chirp function $\alpha$ (24) so as to include to some extent the effect of the collective force and maintain the support of the beam density centered in the grid used to represent the beam density. Implementing this poses no problem in principle.

A further problem could appear if during its evolution the correlation function $\alpha$ became multivalued or had an infinite slope, making the coordinate transformation (28) and (29) ill defined (e.g. see Fig. 6 in [29]). An obvious solution would be to distinguish between the regions of phase space where the transformation can be uniquely defined and apply the method of Sec. V to each region separately. It remains to be seen whether this prescription would be practical and efficient.

\section{ACKNOWLEDGMENTS}

Discussions with and comments from G. Bassi, J. Ellison, P. Emma, B. Fawley, and Z. Huang are gratefully acknowledged. This work was supported by Department of Energy Contracts No. DE-AC02-05CH11231 and No. DEAC02-76SF00515.

\section{APPENDIX}

The main sources of machine impedance relevant for the microbunching instability are space-charge and CSR. RF structure wakefields can be very significant but are generally effective on a length scale longer than the one relevant for microbunching. For the examples shown in this paper we used the following model $[3,30]$ of space-charge impedance (per unit length):

$$
\hat{Z}(k)=\left.\frac{i Z_{0}}{\pi \gamma r_{b}} \frac{1-x K_{1}(x)}{x}\right|_{x=k r_{b} / \gamma},
$$

where $K_{1}(x)$ is a modified Bessel function and $Z_{0}=$ $120 \pi \Omega$, the vacuum impedance. This formula applies to a bunch with transversally uniform density and circular cross section of radius $r_{b}$ in free space and yields the electric field on the beam axis. This expression is valid for wavelengths small compared to $b / \gamma$ [22], where $b$ is the radius of the vacuum chamber $(\gamma$ is the relativistic factor).

For CSR we used the impedance model [2] for radiation in free space,

$$
\hat{Z}(k)=Z_{0} \frac{\Gamma(2 / 3)}{3^{1 / 3} 4 \pi R}(\sqrt{3}+i)(k R)^{1 / 3},
$$

where $\Gamma$ is the Euler function. Because we are mostly interested in the evolution of high frequency components of the bunch spectrum, this is adequate for our study: shielding from the vacuum chamber [11] becomes noticeable for wavelengths longer than about $b(2 b / R)^{1 / 2}$. For the FERMI chicane parameters $(b \simeq 4 \mathrm{~mm}$ and radius of curvature $R \simeq 7.4 \mathrm{~m}$ ), this number is about $130 \mu \mathrm{m}$-larger than wavelengths of interest for microbunching. An additional approximation is to neglect transient effects at dipole ends, as the impedance (A2) properly applies to particles in uniform motion on a circular orbits: the criterion [1] for the validity of (A2), $\lambda \leq R \phi^{3} / 24$, where $\phi$ is the dipole bend angle, yields a $100 \mu \mathrm{m}$ critical wavelength (for $R \simeq 7.4 \mathrm{~m}$ and $\phi=70 \mathrm{mrad}$ ).

The expressions (A1) and (A2) are understood to be valid for $k>0$. For negative wave numbers, the complete expressions are recovered by the rule $Z(-k)=Z^{*}(k)$.

[1] E. L. Saldin, E. A. Schneidmiller, and M. V. Yurkov, Nucl. Instrum. Methods Phys. Res., Sect. A 483, 516 (2002).

[2] S. Heifets, G. Stupakov, and S. Krinsky, Phys. Rev. ST Accel. Beams 5, 064401 (2002).

[3] Z. Huang and K.-J. Kim, Phys. Rev. ST Accel. Beams 5, 074401 (2002).

[4] Z. Huang, M. Borland, P. Emma, J. Wu, C. Limborg, G. Stupakov, and J. Welch, Phys. Rev. ST Accel. Beams 7, 074401 (2004).

[5] C. Bohn, in Advanced Accelerator Concepts: Tenth Workshop, AIP Conf. Proc. No. 647 (AIP, New York, 2002), pp. 81-95.

[6] C.Z. Cheng and G. Knorr, J. Comput. Phys. 22, 330 (1976).

[7] O. Boine-Frankenheim, I. Hofmann, and G. Rumolo, Phys. Rev. Lett. 82, 3256 (1999).

[8] E. Sonnendrücker, J. Roche, P. Bertrand, and A. Ghizzo, J. Comput. Phys. 149, 201 (1999). 
[9] R. Warnock and J. Ellison, in Proceedings of the 2nd ICFA Advanced Workshop on Physics of High Brightness Beams, Los Angeles, 1999 (World Scientific, Singapore, 2000); also available as SLAC-PUB-8404, 2000.

[10] M. Venturini and R. Warnock, Phys. Rev. Lett. 89, 224802 (2002).

[11] M. Venturini, R. Warnock, R. Ruth, and J. A. Ellison, Phys. Rev. ST Accel. Beams 8, 014202 (2005).

[12] R. Warnock, Nucl. Instrum. Methods Phys. Res., Sect. A 561, 186 (2006).

[13] R. Warnock, G. Bassi, and J. Ellison, Nucl. Instrum. Methods Phys. Res., Sect. A 558, 85 (2006).

[14] A. Sobol, Ph.D. dissertation, Mathematics, University of New Mexico, 2006.

[15] N. Crouseilles, G. Latu, and E. Sonnendrücker, Research report INRIA Lorraine 5926 (http://hal.inria.fr/inria00078455).

[16] M. Gutnic, G. Latu, and E. Sonnendrücker, in Proceedings of the 16th International Symposium on Heavy Ion Fusion, Saint Malo, France [Nucl. Instrum. Methods Phys. Res., Sect. A (to be published)].

[17] M. Gutnic, M. Haefele, and E. Sonnendrücker, Nucl. Instrum. Methods Phys. Res., Sect. A 558, 159 (2006); M. Mehrenberger, E. Violard, O. Hoenen, M. Campos Pinto, and E. Sonnendrücker, ibid. 558, 188 (2006).

[18] J. Behrens and A. Iske, Comput. Math. Appl. 43, 319 (2002).

[19] Throughout this paper $p$ will always denote an energy, not a momentum.

[20] R. Warnock, R. Ruth, M. Venturini, and J. A. Ellison, Phys. Rev. ST Accel. Beams 8, 014402 (2005).
[21] R. Warnock and M. Venturini, Proceedings of the Particle Accelerator Conference, 2003 (IEEE, Piscataway, NJ, 2003), p. 3144.

[22] A. Chao, Physics of Collective Beam Instabilities in High Energy Acceleratos (Wiley, New York, 1993).

[23] We use $\mathrm{a}^{\wedge}$ to remind ourselves that $\hat{Z}$ is an impedance per unit length.

[24] R. Ruth, in Nonlinear Dynamics Aspects of Beam Dynamcis, Lecture Notes in Physics (Springer-Verlag, Berlin, 1986).

[25] This algorithm specifically applies to bunch compressors; in an accelerating structure advancement of the beam density is much simpler as we only need to apply energy kicks and items (i) through (iv) can be skipped.

[26] C. Bocchetta et al., Proceedings of the FEL Conference 2006, Berlin, Germany (unpublished).

[27] Here $\rho_{i}$ and $\rho_{f}$ refer to the beam density in the absence of the sinusoidal modulation.

[28] After completion of this work, we came to the realization that for Gaussian beams this choice may lead to overestimating the space-charge impedance while the expression suggested in Appendix A of [4] $r_{b}=1.7\left(\sigma_{x}+\sigma_{y}\right) / 2$ appears to be a better fit. We will address this point more at length in a forthcoming paper.

[29] K. Bane, Proceedings of the 1991 Particle Accelerator Conference (1991), p. 1800.

[30] J. Rosenzweig, C. Pellegrini, L. Serafini, C. Ternieden, and G. Travish, Nucl. Instrum. Methods Phys. Res., Sect. A 393, 376 (1997). 